\title{
Cladophialophora Bantiana as a Cause of Rare Fungal Brain Abscess
}

\author{
Zafar Ali ${ }^{1}$, Muhammad Usman², Usama Rehman ${ }^{1}$ and Fatima Anwar ${ }^{1}$ \\ ${ }^{1}$ Department of Histopathology, Shifa International Hospital, Islamabad, Pakistan \\ ${ }^{2}$ Department of Microbiology, Shifa International Hospital, Islamabad, Pakistan
}

\begin{abstract}
Cerebral phaeohyphomycosis refers to central nervous system infection caused by dematiaceous molds, which have many genuses. Cladophialophora bantiana is a member of the phylum ascomycota, which is found in soil samples from all over the world. This organism typically infects immunocompromised patients and associated with $70 \%$ mortality rate even after weeks of antifungal agent administration and surgical debridement. Two such cases of fungal brain abscess caused by cladophialophora bantiana were presented here. Both patients presented with complaints of headache, vomiting, drowsiness and impaired cognition. A brain biopsy together with microbiological culture and VITEK 2 helped in reaching to a final diagnosis.
\end{abstract}

Key Words: Cladophialophora bantiana, Dematiaceous, Intracerebral, Phaeohyphomycosis.

How to cite this article: Ali Z, Usman M, Rehman U, Anwar F. Cladophialophora Bantiana as a Cause of Rare Fungal Brain Abscess. J Coll Physicians Surg Pak 2021; 31(09):1114-1116.

\section{INTRODUCTION}

Central nervous system (CNS) infections caused by dematiaceous molds are referred as cerebral phaeohyphomycosis. ${ }^{1}$ Cladophialophora bantiana (C. bantiana) is the most common species associated with $70 \%$ mortality. ${ }^{2}$ Immunocompromised patients are more susceptible. CNS infection usually results from hematogenous spread from lungs. ${ }^{3}$ Radiologically and clinically, it mimics infections such as tuberculosis, toxoplasmosis and brain tumors. Histologically, it is very difficult to differentiate among varioustypes of pigmented fungal species, i.e. chromomycosis and phaeohyphomycosis, as they have darkly pigmented hyphae and spores, or both. So, definite diagnosis requires a sample for microbiological and histopathological evaluation and definite mold identification. Two such cases of CNS infections caused by $C$. bantiana were presented in this case report.

\section{CASE NO. 1:}

A 23-year male presented to Emergency Department (ED) in 2013 with history of fever, vomiting and decreased speech for the last three days. He had past history of meningitis and hydrocephalus for two weeks, for which ventriculoperitoneal (VP) shunt was placed at some outside hospital.

Correspondence to: Dr. Usama Rehman, Department of Histopathology, Shifa International Hospital, Islamabad, Pakistan

E-mail: usama_rhmn@yahoo.com

Received: October 10, 2019; Revised: January 02, 2020; Accepted: January 31, 2020

DOI: https://doi.org/10.29271/jcpsp.2021.09.1114

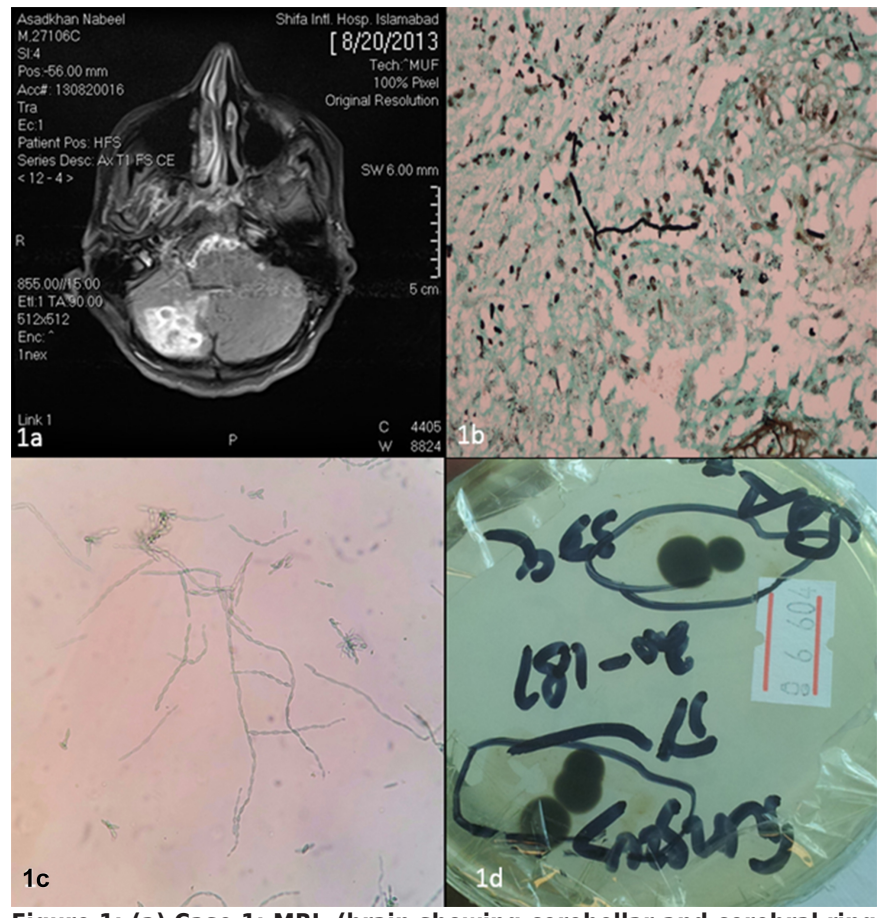

Figure 1: (a) Case 1: MRI (brain showing cerebellar and cerebral ring enhancing lesions. (b) Gomori methenamine silver stain (GMS) special stain highlighting branching hyphae. (c) Lactophenol stain showing delicate hyphae. (d) Culture plate showing black colonies with velvety appearance.

His initial laboratory studies revealed slightly increased total leukocyte counts (TLCS) and erythrocyte sedimentation rate (ESR) of $10 \mathrm{~mm} / 1^{\text {st }} \mathrm{hr}$ and C-reactive protein (CRP) of $1.7 \mathrm{mg} / \mathrm{dl}$. Initial imaging (MRI brain with contrast) revealed multiple nodular/ring enhancing lesions in the supratentorial and infratento- 
rial compartments, along the surface of brain stem, spinal cord, and left thalamus with diffuse leptomeningeal enhancement and meningeal involvement throughout the spine. Radiological findings were suspicious for tuberculous meningitis with brain tuberculomas. He was empirically started on antituberculous therapy (ATT), but his condition deteriorated and craniotomy was performed. Tissue was obtained for both microbiological and histopathological evaluations. Histopathological examination showed necrotising granulomas with pigmented septate hyphae (highlighted by PAS and GMS stains). Gram stain was negative and bacterial cultures showed no growth. Fungal culture showed small, round, 5-10 mm growth after 10 days of incubation. The colony had velvety texture. Lactophenol cotton blue preparation showed dark brown colour septate hyphae with long, sparsely branched conidiophores bearing wavy chains of smooth oval conidia measuring upto 5-10 $\mu \mathrm{m}$ in length. On the basis of typical fungal growth colonies along with VITEK 2 identification, a diagnosis of cladophialophora bantiana was made (Figure 1). Patient was started on intravenousamphotericin B; but despite aggressive treatment, he died after three weeks ofadmission.

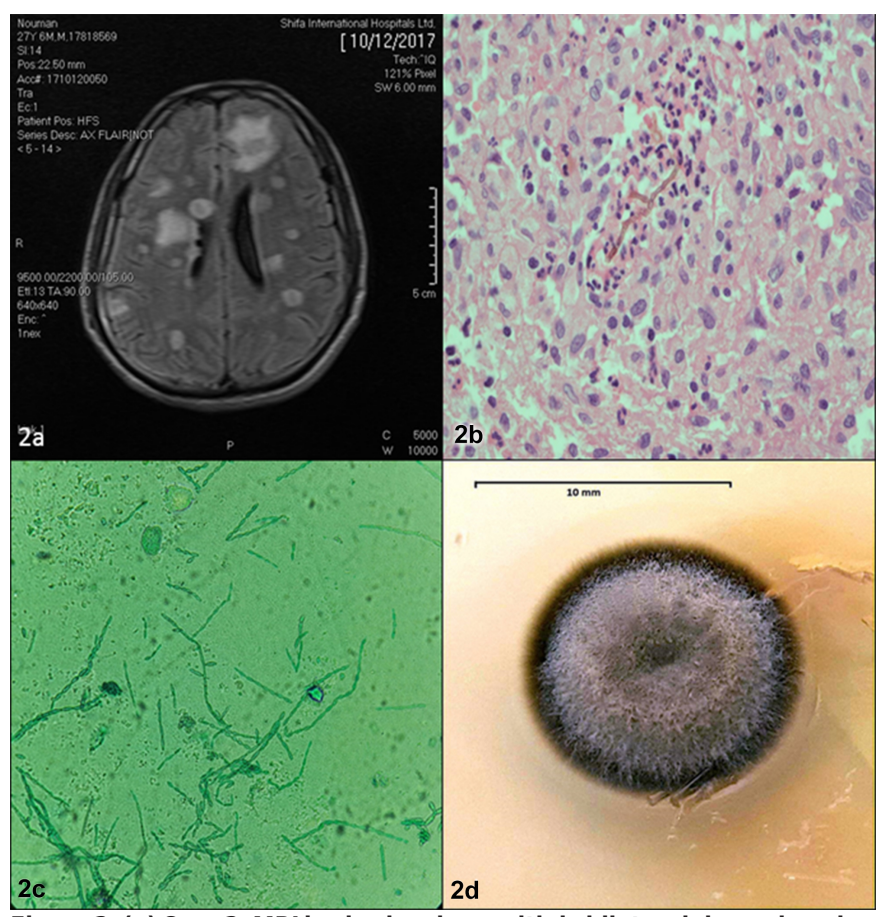

Fiureg 2: (a) Case 2: MRI brain showing multiple bilateral ring enhancing lesions. (b) Hematoxylin and eosin (HE) stain highlighting pigmented branching hyphae. (c) Lactophenol stain showing hyphae. (d) Culture plate showing blackfungus with velvety appearance.

\section{CASE NO. 2:}

A 27-year male presented to this hospital in ED in 2017, with complaints of fever of two weeks duration, and body rash, vomiting, lower limb weakness and headache for five days. At the time of admission, Glasgow coma scale (GCS) was 13/15. Electroencephalogram (EEG) showed moderate encephalopathy. MRI brain with contrast revealed innumerable bilateral ring enhancing intraparenchymal lesions in cerebral, and cerebellar hemispheres with significant perilesional edema and restricted diffusion. Radiological findings were suggestive of tuberculous meningitis. ATT was started. Later, craniotomy was performed and necrotic tissue was sent for both microbiological and histopathological analysis. Biopsy revealed necrotising granulomas with pigmented fungal elements. Fungal culture showed black velvety fungal colonies. Based on these findings, it was classified as C. bantiana species (Figure 2). Systemic antifungal therapy in the form of voriconazole and amphotericin $B$ was started. Patient was discharged on oral antifungal therapy. MRI scan after one month of diagnosis showed much regression of enhancing lesions. The patient died after two months of discharge; and the cause of death could not be ascertained.

\section{DISCUSSION}

CNS infection/abscess with cladophialophora bantiana is rare and mostly fatal. Clinical presentation raises suspicion of malignancy. Men are commonly affected. The first culture-proven case of brain abscess due to this fungus was described in an American patient by Binford in 1952. ${ }^{4}$ Many cases have been reported from the Asian continent, especially India. ${ }^{5}$

This organism is highly neurotropic. The predisposing factors include organ transplantation, glucocorticoid treatment, neutropenia, diabetes mellitus, direct inoculation, ocular injury, intravenous drug abuse, etc. which causes cerebral abscesses rather than meningitis. ${ }^{6}$ This fungus lives in soil or on vegetations. ${ }^{7}$ Its mode of entry is not well understood; ${ }^{8}$ however, Revankar et.al ${ }^{9}$ suggested entry through inhalation of spores followed by hematogenous dissemination to the brain. Puncture wounds rarely can also lead to hematogenous spread. One hypothesis pertaining to its brain invasion properties is that melanin within the fungus shows affinity to receptors on the blood-brain barrier, allowing the fungus to penetrate into the parenchyma. Both the patients were immunocompetent and students by profession. They had no history of trauma and their chest radiographs were normal. Both did not have any previous history of seizures and presented with short history of illness. Radiological examinations revealed extensive bilateral CNS lesions, which mimicked tuberculosis.

Histopathologically, the glial tissue shows necrosis and granulation tissue formation. Presence of multinucleated giant cells and pigment raises the possibility of some obscure fungal species. Special stains like PAS and GMS are very helpful in identification of this group of organisms. Moreover, C. bantiana has brown to black colour, pigmented hyphae. Presence of multinucleated giant cells and necrotic slough raises the possibility of other differentials like tuberculosis and toxoplasmosis, which are more common in ourpopulation.

Limited data is available regarding the treatment of disease due to its rarity and lack of prospective trials. Treatment strategies include combination of surgery and systemic antifungal therapy. It is imperative not to delay treatment and to obtain a tissue sample of the lesion as soon as possible. Surgically, the aim should be to remove as much diseased tissue as possible and send specimen for microbiology as well as histopathology. 
Voriconazole has good CNS penetration and consistent potent activity. ${ }^{8}$ Due to this dangerous biological agent, it has been added to biosafety level 2, and also level 3 in some parts of the world. The laboratory personnel have to take extra precautionary measures while dealing with this agent. ${ }^{7}$ Both patients did not survive due to grim nature of disease even after extensive treatment.

In summary, cladophialophora bantiana should be considered whenever a pigmented fungus is seen in the brain. Early radiological intervention can be fruitful. Tissue diagnosis is imperative to avoid unnecessary treatment. Collaboration between histopathology and microbiology is required for acquiring the definite diagnosis.

\section{PATIENTS' CONSENT:}

Because this study was retrospective, the condition of patients' consent was waived.

\section{CONFLICT OF INTEREST:}

The authors declared no conflict of interest.

\section{AUTHORS' CONTRIBUTION:}

ZA: Carried out the conception and design of the research, drafted the manuscript.

MU: Drafted a part of manuscript.

UR: Composed and designed the manuscript.

FA: Assisted in drafting the data.

All authors read and approved the final manuscript.

\section{REFERENCES}

1. Jung NY, Kim E. Cerebral phaeohyphomycosis: A rare cause of brain abscess. J Korean Neurosurg Soc 2014; 56(5): 444-7. doi: 10.3340/ jkns.2014.56.5.444.

2. Ahmad M, Jacobs D, Wu HH, Wolk DM, Kazmi SA, Jaramillo
C, et al. Cladophialophora bantiana: A rare intracerebral fungal abscess-case series and review of literature. Surg J (N Y) 2017; 3(02):e62-8. doi: 10.1055/s-0037-1598248.

3. Desmet S, Smets L, Lagrou K, Derdelinckx I, Neyt J, Maertens J, et al. Cladophialophora bantiana osteomyelitis in a renal transplant patient. Med Mycol Case Rep 2016; 12:17-20. doi: 10.1016/j.mmcr.2016.07.002.

4. Binford $\mathrm{CH}$, Thompson RK, Gorham ME. Mycotic brain abscess due to cladosporium trichoides, a new species. Am J Clin Pathol 1952; 22(6):535-42. doi: 10.1093/ajcp/ 22.6.535.

5. Khader A, Ambooken B, Binitha MP, Francis S, Kuttiyil AK, Sureshan DN. Disseminated cutaneous phaeohyphomycosis due to Cladophialophora bantiana.Indian J Dermatol Venereol Leprol 2015; 81(5):491. doi: 10.4103/0378-6323. 162333.

6. Aljuboori Z, Hruska R, Yaseen A, Arnold F, Wojda B, Nauta H. Fungal brain abscess caused by "black mold" (cladophialophora bantiana): A case report of successful treatment with an emphasis on how fungal brain abscess may be different from bacterial brain abscess. Surgical Neurol Int 2017; 8:46. doi: 10.4103/sni.sni_448_16.

7. Kantarcioglu AS, Guarro J, de Hoog GS, Apaydin H, Kiraz N, Balkan II, et al. A case of central nervous system infection due to cladophialophora bantiana. Rev Iberoam Micol 2016; 33(4):237-41. doi: 10.1016/j.riam.2016.01.004.

8. Nau R, Sörgel F, Eiffert H. Penetration of drugs through the blood-cerebrospinal fluid/blood-brain barrier for treatment of central nervous system infections. Clin Microbiol Rev 2010 Oct;23(4):858-83. doi: http://doi.org/10.1128/ CMR.00007-10.

9. Revankar SG, Sutton DA, Rinaldi MG. Primary central nervous system phaeohyphomycosis: A review of 101 cases. Clin Infect Dis 2004; 38(2):206-16. doi: 10.1086/ 380635 . 